\title{
Gastrointestinal and urinary tract pathogenic infections among HIV seropositive patients at the Komfo Anokye Teaching Hospital in Ghana
}

\author{
Yaw Agyekum Boaitey ${ }^{1}$, Bernard Nkrumah ${ }^{2 *}$, Ali Idriss ${ }^{3}$ and Samuel Crowther Kofi Tay ${ }^{4}$
}

\begin{abstract}
Background: Gastrointestinal and urinary tract pathogenic infections are aggravating the incidence and progression of the Human Immunodeficiency Virus (HIV) infection into Acquired Immune Deficiency Syndrome (AIDS) more especially in the developing countries. This study was conducted to assess the common gastrointestinal and urinary infections among HIV/AIDS patients at the Komfo Anokye Teaching Hospital (KATH) in Ghana between April and December 2008.

Findings: This work reports on gastrointestinal and urinary tract pathogenic infections among 500 HIV seropositive and 300 HIV seronegative patients. There was a 35\% (175/500) prevalence of intestinal parasites among HIV seropositive patients compared to 4.3\% (13/300) in HIV seronegative patients. Giardia lamblia and Cryptosporidium accounted for 19\% (95/500) and 14\% (70/500) respectively, while Schistosoma mansoni, Strongyloides stercoralis and hookworm together accounted for 2\% (10/500) of intestinal parasitic infections among the HIV seropositive patients. There was no significant difference $(p>0.05)$ in urinary parasitic infection between HIV seropositive 1\% $(2 / 500)$ and seronegative patients $0.7 \%(2 / 300)$. Most, 60 (86\%) out of 70 , of the urinary tract infection among the HIV seropositive patients was due to bacteria with E. coli being the most predominant isolate, 28 (47\%) out of 60 . There was no significant difference in infections based on age and gender.
\end{abstract}

Conclusion: G. lamblia and Cryptosporidium were the most common gastrointestinal parasites detected while bacteria accounted for majority of the urinary tract infections among the HIV seropositive patients at the hospital.

Keywords: HIV/AIDS, Giardia lamblia, Cryptosporidium, Parasitic infection

\section{Background}

Parasitic infections caused by helminth and protozoans are among the most prevalent infections in humans worldwide especially, in the tropical and sub tropical areas of the developing world. The problems posed by parasitic infections have long been recognized and the subject has thus merited increasing importance over the years [1]. Several outbreaks of diarrheal diseases caused by these parasites have been reported during the last decade $[2,3]$ and are estimated to cause over one million deaths each year [4]. Epidemiological surveys have shown that poor sanitation and inappropriate environmental conditions coupled with indiscriminate

\footnotetext{
*Correspondence: skrakyo@yahoo.com

${ }^{2}$ Kumasi Centre for Collaborative Research in Tropical Medicine, Kumasi, Ghana

Full list of author information is available at the end of the article
}

defecation, geophagy and contamination of water bodies in these countries are the most important predisposing factors [5]. Studies have shown higher rates of parasitic infections in HIV/AIDS positive than negative subjects [6]. HIV/AIDS compromises the immune system of the body thus patients with HIV/AIDS are threatened by a great number of diseases such as those caused by different kinds of biological agents including bacterial, fungal, viral, protozoa and helminth parasitic pathogens. In such patients, opportunistic parasitic gastrointestinal infections presents in the form of severe diarrhea which profoundly compromises the absorptive function of the small intestine, leading to significant mortality [7]. Helminth parasitic infections also cause chronic immune activation [8-10] which has been shown to increase host susceptibility, thereby promoting HIV/AIDS infection and disease progression [11,12]. Infections with the

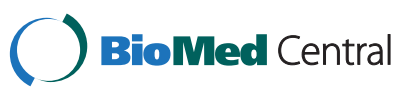


major human gastrointestinal parasites have been shown to be rampant in most parts of Africa and the prevalence of these infections varies from country to country [1316] with Ghana being no exception [17]. Studies conducted in the Ashanti Region of Ghana have shown the presence of gastrointestinal infections among both children and adults [18-21]. However, information regarding the magnitude of opportunistic parasitic infections among HIV/AIDS positive individuals is scarce and not well studied. This study was therefore undertaken to contribute to the knowledge of the occurrence of gastrointestinal and urinary tract infections among HIV/AIDS positive and negative patients at Komfo Anokye Teaching Hospital (KATH) in Kumasi, Ghana.

\section{Methods}

\section{Study design}

This was a hospital based cross-sectional study of HIV seropositive and negative adults [22] presenting to the Voluntary Counseling and Testing Unit and Chest Clinic of the Komfo Anokye Teaching Hospital (KATH).

\section{Study site}

The study was performed at the Voluntary Counseling and Testing Unit and Chest Clinic of KATH, Ghana from April to December 2008. KATH is approximately a thousand bed tertiary medical facility located in Kumasi, Ashanti Region, the confluence of the transportation network in the central part of Ghana. Its position makes it the most accessible tertiary and referral medical facility in Ghana attending to a population of over 4.4 million in the Ashanti region and beyond [23].

\section{Ethical approval}

The study protocol was approved by the Committee on Human Research, Publications and Ethics, School of Medical Sciences, Kwame Nkrumah University of Science and Technology in collaboration with KATH management.

\section{Stool collection}

The parasitology laboratory of KATH is a well established unit that operates by the internationally accepted Good Laboratory Practice (GLP). Stool samples were collected in accordance with WHO guidelines on the collection of faecal samples [24] and as outlined previously by Nkrumah et al. [20].

\section{Urine collection}

Urine samples were collected as previously described by Cheesbrough [6].

\section{Specimen processing and examination HIV testing}

HIV testing for study subjects was conducted at the VCT centre and the serology department of the hospital. Testing was done using the national algorithm. Briefly, all tests were done using the First Response HIV Card 1-2.0 (PMC Medical India Pvt Ltd). Positive tests were confirmed with OraQuick ADVANCE Rapid HIV-1/2 (OraSure Technologies, Inc.). Enzyme-Linked Immunosorbent Assay (ELISA) was used as a tie breaker. All tests were performed as outlined in the manufactures' manual.

\section{Stool samples \\ Direct microscopy}

Direct microscopy of the smear in saline $(0.90 \% \mathrm{w} / \mathrm{v}$ $\mathrm{NaCl}$ solution) and Lugol's iodine was performed for the detection of parasites (trophozoites, cysts, egg and larvae) as previously described [25]. In cases where parasite species identification was not conclusive the attendant microscopist requested a senior microscopist to confirm the results. Where no consensus was reached, the formol-ether concentration method was employed. The average time between sample collection and processing was 30 minutes.

\section{Formol-ether concentration method}

The formol-ether concentration method was performed and examined as previously described by the WHO [24] and Ayeh-Kumi et al. [25]. The average time between sample collection and processing was 50 minutes.

\section{Modified Ziehl Neelsen (ZN) staining method}

The modified ZN staining method was used for the identification of Cryptosporidium. This method was performed and examined as earlier described by AyehKumi and his group [25].

\section{Urine samples}

\section{Microscopic examination-urine sedimentation method}

Urine samples were processed and examined as described by Cheesbrough [6]. Urine samples with pus cells $>5$ /HPF were cultured. The average turnaround time was 20 minutes.

\section{Culture process}

Urine samples were cultured using a standard loop calibrated to hold $0.01 \mathrm{ml}$ of urine onto Cysteine Lactose Electrolyte Deficient (CLED) agar. Inoculated plates were incubated at $37^{\circ} \mathrm{C}$ aerobically overnight. After overnight incubation the plates were read and the growths were identified based on the growth characteristics on the inoculated media. 


\section{Colony counts}

Colonies were counted on CLED and multiplied by the loop volume. A bacterial count of $1 \times 10^{5}$ per ml was considered significant for UTI while counts less than $1 \times 10^{5}$ per $\mathrm{ml}$ were considered as no significant bacterial growth [6].

\section{Bacterial identification}

Bacterial isolates were identified using conventional biochemical methods including urease and indole production, citrate utilization, hydrogen sulphide, gas production and fermentation of sugars. The biochemical media used included Simon's Citrate medium, Urea and Triple Sugar Iron agar (TSI). Catalase and coagulase tests were performed for all staphylococci organisms.

\section{Antimicrobial sensitivity testing}

Antimicrobial susceptibility test were performed using Kirby-Bauer disc diffusion method. The isolates from this study were tested against the following antibiotics: Ampicillin $(10 \mu \mathrm{g})$, Cefuroxime $(30 \mu \mathrm{g})$, Cotrimoxazole $(25 \mu \mathrm{g})$, Ciprofloxacin $(5 \mu \mathrm{g})$, Gentamicin $(10 \mu \mathrm{g})$, Tetracycline $(30 \mu \mathrm{g})$, Nalidixic acid $(30 \mu \mathrm{g})$ and Nitrofurantoin $(300 \mu \mathrm{g})$. Zone sizes were measured according to CLSI standards. The average turnaround time was 2 days.

\section{Findings}

A total of 800 subjects were examined of which 500 (62.5\%) out of 800 were HIV seropositive subjects. Females constituted $52.7 \%(419 / 800)$ of the total study population. Out of 500 HIV seropositive patients, 258 (51.6\%) presented with diarrhea or fever. Out of these 258, 149 (57.8\%), 60 (23.3\%) and 10 (9.2\%) were positive for intestinal parasites, bacteruria or both respectively. Subjects within 21-30 years constituted the highest age group of the study population $38.4 \%$ (307/800) (Table 1). Out of 500 HIV seropositive patients, 365 (73\%) and out of 300 HIV seronegative patients, 217 (72.3\%) were recruited within the wet season respectively. There were more symptomatic seropositive subjects in the wet season; $61.4 \%$ (224/365), than in the dry season; $25.2 \%$ (34/
135), with more parasites being isolated from symptomatic subjects within the wet season 87.5\% (196/224).

Gastrointestinal infection was found in 175 (35\%) out of 500 HIV seropositive subjects, and only in 13 (4.3\%) out of 300 HIV seronegative subjects, with a statistically significant difference $(\mathrm{p}<0.05)$. However, there was no significant difference in urinary infection among 5 (1.0\%) out of 500 HIV seropositive subjects and $2(0.7 \%)$ out of 300 seronegative subjects $(\mathrm{p}>0.05)$ (Table 2). Among the various age groups, there was no significant difference $(\mathrm{p}>0.05)$ in urinary parasitic infection rate. Infection with G. lamblia (19\%) and Cryptosporidium (14\%) were statistically higher in HIV seropositive subjects $(\mathrm{p}<0.05) \quad$ (Table 3$)$. Out of 70 HIV seropositive patients suspected of urinary tract infection, $86 \%$ had bacteruria while $28 \%$ was recorded for HIV seronegative patients $(n=18)$.

One of the most important clinical manifestations in HIV/AIDS patients is diarrhea. This might be caused by various etiological agents including parasitic coccidian and flagellates, bacteria and fungi. Most of these agents can cause life threatening chronic watery diarrhea, weight loss and malabsorption. This makes the persons' sense of well being and ability to carry out daily activities profoundly impaired [26]. A prevalence of 35\% of intestinal parasites among the HIV seropositive patients in this study corresponds with reports from other African countries. Awole et al. [16] reported a prevalence rate of 44.8\% among HIV infected patients in Ethiopia while Agi [4] recorded $33.6 \%$ in Sagbanna community of Niger-Delta in Nigeria. These high prevalence rates are consistent with the fact that, one of the factors that predispose people to parasitic infection is the presence of co-existing disease (example HIV infection) or other conditions which reduces natural immune responses [6]. The low urinary infection rate $(1.0 \%)$ and the high prevalence of bacterial infection (86\%) among the HIV seropositive patients suggest that suspected urinary tract infection (urine samples with pus cells $>5 / \mathrm{HPF}$ ) among these patients are mostly due to bacterial infections but not of parasitic source. E coli and Staph aureus were the most isolated organisms. The antibiotic sensitivity

Table 1 Overall infection rates by age group of the studied populations

\begin{tabular}{|c|c|c|c|c|c|c|c|c|c|c|c|c|c|c|c|c|c|c|}
\hline \multirow{2}{*}{$\begin{array}{l}\text { Age } \\
\text { groups }\end{array}$} & \multicolumn{3}{|c|}{ Males } & \multicolumn{3}{|c|}{ Females } & \multicolumn{3}{|c|}{ Total } & \multicolumn{3}{|c|}{ Males } & \multicolumn{3}{|c|}{ Females } & \multicolumn{3}{|c|}{ Total } \\
\hline & No & Parasite + & $(\%)$ & No & Parasite + & $(\%)$ & No & Parasite + & $(\%)$ & No. & Parasite + & $(\%)$ & No & Parasite + & (\%) & No & Parasite + & (\%) \\
\hline$\leq 20$ & 23 & 4 & 17.3 & 26 & 6 & 23 & 49 & 10 & 20.4 & 23 & 0 & 0 & 26 & 0 & 0 & 49 & 0 & 0 \\
\hline $21-30$ & 152 & 42 & 27.6 & 155 & 40 & 25.8 & 307 & 82 & 26.7 & 152 & 2 & 1.3 & 155 & 1 & 0.6 & 307 & 3 & 0.9 \\
\hline $31-40$ & 144 & 30 & 20.8 & 154 & 37 & 24.2 & 298 & 67 & 22.5 & 144 & 1 & 0.7 & 154 & 2 & 1.2 & 298 & 3 & 1 \\
\hline $41-50$ & 60 & 11 & 18.3 & 70 & 15 & 21.4 & 130 & 26 & 20 & 60 & 0 & 0 & 70 & 1 & 1.4 & 130 & 1 & 0.8 \\
\hline$>50$ & 2 & - & 0 & 14 & 3 & 21.4 & 16 & 3 & 18.8 & 2 & 0 & 0 & 14 & 0 & 0 & 16 & 0 & 0 \\
\hline Total & 381 & 87 & 22.8 & 419 & 101 & 24.1 & 800 & 188 & 23.5 & 381 & 3 & 0.8 & 419 & 4 & 0.9 & 800 & 7 & 0.9 \\
\hline
\end{tabular}

Gastrointestinal infection rate Urinary parasitic infection rate. 
Table 2 Overall parasite infection according to sex of the studied populations

\begin{tabular}{|c|c|c|c|c|c|c|c|c|c|c|c|c|c|c|c|c|c|c|}
\hline \multirow{2}{*}{$\begin{array}{l}\text { Patient } \\
\text { groups }\end{array}$} & \multicolumn{3}{|c|}{ Males } & \multicolumn{3}{|c|}{ Females } & \multicolumn{4}{|c|}{ Total } & \multicolumn{3}{|c|}{ Males } & \multicolumn{3}{|c|}{ Females } & \multicolumn{2}{|l|}{ Total } \\
\hline & No. & Parasite + & $(\%)$ & No. & Parasite + & $(\%)$ & No. & Parasite + & $(\%)$ & No. & Parasite + & $(\%)$ & No. & Parasite + & (\%) & No. & Parasite + & $(\%)$ \\
\hline $\begin{array}{l}\text { HIV + } \\
\text { patients }\end{array}$ & 241 & 82 & 34 & 259 & 93 & 35.9 & 500 & 175 & 35 & 241 & 2 & 0.8 & 259 & 3 & 1.1 & 500 & 5 & 1 \\
\hline $\begin{array}{l}\text { HIV - } \\
\text { patients }\end{array}$ & 140 & 5 & 3.6 & 160 & 8 & 5 & 300 & 13 & 4.3 & 140 & 1 & 0.7 & 160 & 1 & 0.6 & 300 & 2 & 0.7 \\
\hline Total & 381 & 87 & 22.8 & 419 & 101 & 24.1 & 800 & 188 & 23.5 & 381 & 3 & 0.8 & 419 & 4 & 0.9 & 800 & 7 & 0.9 \\
\hline
\end{tabular}

Gastrointestinal parasitic infection.

Urinary parasitic infection.

patterns from this study showed that most of the E. coli isolated was sensitive to nitrofurantoin, gentamicin, cefuroxime and ciprofloxacin. This sensitivity pattern is consistent with a study earlier conducted by Turpin and his colleagues within the same hospital [27] and also consistent to results obtained by Obirikorang and friends in another study conducted within the study area [28]. This low prevalence of urinary parasitic infection is consistent with a 6 year retrospective and prospective study of urinary parasitic infections at KATH which also found that these parasites are less commonly found in routine urine analysis (unpublished data). The even distribution of the parasitic infections in both sexes as well as, among the various age groups suggested that sex and age were not predetermining factors for parasitic infections. The result of this study also revealed a trend in the occurrence of specific intestinal parasites in HIV seropositive patients: G. lamblia (19\%), Cryptosporidium (14\%), Hookworm (1\%), Schistosoma mansoni (0.6\%) and Strongyloides stercoralis (0.4\%). The results of this study disagreed with those of Lindo et al. [29] who found Trichuris trichuria (21.1\%), Hookworm (17.3\%) and Strongyloides stercoralis (7.7\%) in stool samples of HIV infected individuals in San Pedro Sula, Hunduras in Central America. Makri et al. [30] observed that AIDS patients made up a substantial proportion of reported cryptosporidiosis cases. The high prevalence of Cryptosporidium in this study confirmed this parasite as one of the usual opportunistic pathogens commonly associated with HIV infection [6] even though a previous study at the University of Ghana Medical School reported the prevalence of Cryptosporidium species in both HIV seropositive and seronegative individuals with no significant statistical association between cryptosporidiosis and HIV infection [7]. S. stercoralis prevalence (0.4\%) among the HIV seropositive patients in this study was not significant but very important as it can assume a role of extreme gravity due to the immune state of these individuals. This is because this parasitic helminth matures very fast and is capable of autoinfection; the larvae can easily cross the blood brain barrier and then invade the brain tissues [31] which can be very fatal. Apart from S. stercoralis, low prevalence rates were recorded for hookworm (1\%) and S. mansoni (0.6\%). These low rates could be as a result of three main reasons: (1) the effective counseling and continuous education on hygienic practices for HIV seropositive individuals, (2) the high prevalence of G. lamblia and Cryptosporidium sp. compared to hookworm and S. mansoni among HIV/ AIDS patients within the study area, (3) the complex mode of infection of hookworm and S. mansoni compared to that of G. lamblia and Cryptosporidium sp. Even though the residential areas of the patients were not investigated these parasites are associated with poor environmental sanitation and lack of good water supply [5]. There was also a relationship between the seasonality, symptomatology and parasitemia.

\section{Conclusion}

The results of the study revealed G. lamblia and Cryptosporidium as the commonest intestinal parasitic agents among HIV seropositive patients at the Komfo Anokye Teaching Hospital. Most of the urinary tract infections in these patients were due to bacteria than parasites while age and sex of patients played no role in the prevalence of these infections. Techniques such as modified

Table 3 Gastrointestinal parasites among HIV seropositive and negative subjects

\begin{tabular}{|c|c|c|c|c|c|c|}
\hline Parasites & HIV positive $(n=500)$ & $\%$ & HIV negative $(n=300)$ & $\%$ & Total $(n=800)$ & $\%$ \\
\hline Schistosoma masoni & 3 & 0.6 & 2 & 0.7 & 5 & 0.6 \\
\hline Giardia lamblia & 95 & 19 & 6 & 2 & 101 & 12.6 \\
\hline Stronglyloides stercolaris & 2 & 0.4 & 1 & 0.3 & 3 & 0.4 \\
\hline Hookworm & 5 & 1 & 2 & 0.7 & 7 & 0.9 \\
\hline Cryptosporidium sp. & 70 & 14 & 2 & 0.7 & 72 & 9 \\
\hline Total & 175 & 35 & 13 & 4.3 & 188 & 23.5 \\
\hline
\end{tabular}


Ziehl Neelsen stain should be considered for clinically suspected or symptomatic HIV/AIDS patients so that parasites such as Cryptosporidium which are common in these patients will not be missed.

\section{Abbreviations}

WHO: World Health Organization; UTI: Urinary Tract Infection; HIV: Human Immunodeficient Virus; AIDS: Acquired Immune Deficiency Syndrome; KATH: Komfo Anokye Teaching Hospital; HPF: High Power Field; CLSI: Clinical and Laboratory Standards Institute; TSI: Triple Sugar Iron.

\section{Competing interests}

The authors declare that they have no competing interest.

\section{Authors' contributions}

YAB planned and designed the study protocol, carried out the study and contributed to the writing of the manuscript. SCKT supervised the study and contributed to the writing of the manuscript, Al contributed to the study and writing of the manuscript and BN contributed to the study and headed the writing of the manuscript. All the authors have read and approved the manuscript.

\section{Acknowledgements}

We are thankful to all the study participants for taking part in the study. We are also thankful to the staff of the Microbiology Department, KATH for their support during the study.

\section{Author details}

${ }^{1}$ Microbiology Department, Komfo Anokye Teaching Hospital, Kumasi, Ghana. ${ }^{2}$ Kumasi Centre for Collaborative Research in Tropical Medicine, Kumasi, Ghana. ${ }^{3}$ Malaria Research Centre, Agogo, Asante Akim North, Ghana. ${ }^{4}$ Microbiology Department, Kwame Nkrumah University of Science and Technology, Kumasi, Ghana.

Received: 19 February 2012 Accepted: 14 August 2012

Published: 21 August 2012

\section{References}

1. World Health Organization: Intestinal protozoa and helminth infection: WHO technical report series. Geneva: World Health Organization; 1981:666.

2. Kingsbury DT, Gerald EW: The National Medical Series for Independent Study, Microbiol, Volume 9. 2nd edition: Harwal Pub Co; 1990:274.

3. Esipisu I: Integrating the fight against malaria with HIV/AIDS and Diarrhoea. Afr Media Mal Res Net 2010, 5:27-28.

4. Agi PI: Pattern of infection of intestinal parasites in Sagbana Community of the Niger Delta, Nigeria. West Afr J Med 1995, 14(1):39-42.

5. Brooker S, Hotez PJ, Bundy DA: Hookworm-related anaemia among pregnant women: Asystematic review. PLoS Neg/ Trop Dis 2008, 2(9):e291.

6. Cheesbrough M: District Laboratory Practice in Tropical, Parts 1 and 2. 1999:183-189.

7. Adjei A, Lartey M, Bosompem KM: Cryptosporidium oocysts in Ghanaian AIDS patients with diarrhoea. East Afr Med J 2003, 30:369-372.

8. Bentwich Z, Kalinkovich A, Weisman Z: Immune activation is a dorminant factor in the pathogenesis of Africa AIDS. Immunol Today 1995, 16:187-191.

9. Ntigwa T, Ndongmo CB, Zekeng L, Same Ekobo A: Parastic infections in HIV infected patients and correlations with the immune status in Cameroon. Int Conf AIDS 2000, 9-14:13. abstract no. MoPeB2275.

10. Grossman Z, Meier-Schellersheim M, Sousa AR, Victorino RM, Paul WE: CD4+ T-cell depletion in HIV infection: are we closer to understanding the cause. Nat Med 2002, 8:319-323.

11. Shapiro-Nahor OA, Kalinkovich A, Weisman Z, Greenberg Z, Nahmias J, Shapiro M, Panet A, Bentwich Z: Increased susceptibility to HIV-1 infection of peripheral blood mononuclear cells from chronic immune-activated individuals. AIDS 1998, 12:1731-1733.

12. Secor WE, Shah A, Mwinzi PM, Ndenga BA, Watta CO, Karanja DM: Increased density of human immunodeficiency virus type 1 co-receptors CCRS and CXCR4 on the surface of CD4(+) T cells and monocytes of patients with Schistosoma mansoni infection. Infect Immun 2004, 71:6668-6671.
13. Hailemariam G, Kassu A, Abebe G, Abate E, Damte D, Mekonnen E, Ota F: Intestinal parasitic infections in HIV/AIDS and HIV seronegative individuals in a teaching hospital, Ethiopia. Jpn J Infect Dis 2004, 57(2):41-43.

14. Ortega YR, Sterling CR, Gilman RH, Cama VA, Diaz F: Cyclospora species-a new protozoan pathogen of humans. N Eng J Med 1993, 328(18):1308-1312.

15. Mengesha B: Cryptosporidiosis among medical patients with the acquired immunodeficiency syndrome in Tikur Anbessa Teaching Hospital, Ethiopia. East Afr Med J 1994, 71(6):376.

16. Awole M, Gebre-Selassie S, Kassa T, Kibra G: Prevalence of Intestinal Parasites in HIV-Infected adult patients in Southwestern Ethiopia. Ethop J 2003, 17(1):71-78.

17. Adjei A, Lartey M, Adiku TK, Rodrigues O, Renner L, Sifah E, Mensah JD, Akanmori BD, Otchere J, Bentum BK: Cryptosporidium oocysts in Ghanaian AIDS patients with diarrhoea. East Afr Med J 2004, 80(7):369-372.

18. Addy PA, Aikins-Bekoe P: Cryptosporidiosis in diarrhoeal children in Kumasi, Ghana. Lancet 1986, 1(8483):735.

19. Addy PAK, Antepim G, Frimpong EH: Prevalence of pathogenic Escherichia coli and parasites in infants with diarrhoea in Kumasi, Ghana. E Afr Med J 2004, 81(7):353-357.

20. Nkrumah B, Nguah SB: Giardia lamblia: a major parasitic cause of childhood diarrhoea in patients attending a district hospital in Ghana. Parasite \& Vectors 2011, 3:163

21. Acquah SEK: Significance of intestinal protozoan parasites as diarrhoeacausing infectious agents in children presenting to the Agogo Presbyterian Hospital. Kwame Nkrumah University of Science and Technology, Department of Clinical Microbiology, School of Medical Sciences, College of Health Sciences. Kumasi, Ghana: Kwame Nkrumah University of Science and Technology; 2010.

22. Keou FX, Belec L, Esunge PM, Cancre N, Gresenguet G: World Health Organization clinical case definition for AIDS in Africa: an analysis of evaluations. East Afr Med J 1992, 69(10):550.

23. Komfo Anokye Teaching Hospita. http://wwwkathhsporg/aboutus1 php.

24. World Health Organisation: Basic Laboratory methods in medical parasitology. Geveva: World Health Organisation; 1991.

25. Ayeh-Kumi PF, Quarcoo S, Kwakye-Nuako G, Kretchy JP, Osafo-Kantanka A, Mortu S: Prevalence of intestinal parasitic infections among food vendors in Accra, Ghana. Journal of Tropical Medicine \& Parasitology 2009, 32(1):1-8.

26. Zelalem TM, Gemeda A, Andargachew M: Opportunistic and Other Intestinal Parasitic Infections in Aids Patients, HIV Seropositive Healthy Carriers and HIV Seronegative Individuals in Southwest Ethiopia. East Afr J Pub Health 2008, 5(3):169-173.

27. Turpin CA, Minkah B, Danso KA, Frimpong EH: Asymptomatic bacteriuria in pregnant women attending antenatal clinic at komfo anokye teaching hospital, kumasi, ghana. Ghana Med J 2007, 41(1):26.

28. Obirikorang C, Quaye L, Bio FY, Amidu N, Acheampong I, Addo K: Asymptomatic Bacteriuria among Pregnant Women Attending An-tenatal Clinic at the University Hospital, Kumasi, Ghana. Journal of Medical and Biomedical Sciences 2012, 1(1):38-44.

29. Lindo JF, Duban JM, Ager AL, Gourville E, Gagriel HI, Klaskala WK, Baum M, Palma CJ: Intestinal parasitic infections in HIV positive and HIV negative individuals in San Pedro Sula, Hundura. AmJTrop Med Hyg 1998 54(4):431-435.

30. Makri B, Parkin E: Susceptibility in microbial risk assessment. Definitions and research needs. Environ Health Perspect 2000, 198(9):905-909.

31. Carnauba MC, Carnauba D, Gama JA: A typical infection by Strongyliodes stercoralis in patients with AIDS. Int Conf AIDS 1996, 11:296.

\section{doi:10.1186/1756-0500-5-454}

Cite this article as: Boaitey et al:: Gastrointestinal and urinary tract pathogenic infections among HIV seropositive patients at the Komfo Anokye Teaching Hospital in Ghana. BMC Research Notes 2012 5:454. 